\title{
A monoclonal antibody targeting the dimer interface of epidermal growth factor receptor (EGFR)
}

Tujing Guo a,b ${ }^{\text {a }}$ Lin Zhao ${ }^{\mathrm{a}, \mathrm{b}}$, Yawen Zhang ${ }^{\mathrm{a}}$, Guoqiang Liu ${ }^{\mathrm{a}}$, Yuanhang Yao ${ }^{\mathrm{a}}$, and Huangjin $\mathrm{Li}^{\mathrm{a}, *}$

${ }^{a}$ Guangdong Provincial Key Laboratory of Biotechnology Candidate Drug Research, School of Biosciences and Biopharmaceutics, Guangdong Pharmaceutical University, Guangzhou 510006, PR China

${ }^{b}$ These authors equally contributed to this work.

*Corresponding Author

Tel.: +862039352326

E-mail address: $1002304772 @ q q . c o m(H . L i)$ 


\begin{abstract}
Epidermal growth factor receptor (EGFR) is an attractive target for the treatment of epithelial cancers. However, EGFR antagonists have low clinical response rates and frequently induce resistance mainly caused by the hypermutation of the extracellular and intracellular domains and the heterodimerization of EGFR. Dimerization plays a key role in the activation of the EGFR family of receptors. Thus, targeting the highly conserved dimer interface of EGFR maybe an attractive strategy for improving the clinical response of anti-EGFR therapies. In this work, we report a monoclonal antibody (mAb) called Antidimer 5G9 that targets the $\beta$-hairpin within the dimer interface of EGFR, which is $100 \%$ homologous with mouse EGFR. Antidimer 5G9 belongs to the isotype IgG1 and bound with an affinity constant of $1.837 \times 10^{9} \mathrm{~L} / \mathrm{mol}$. Just as designed and expected, Antidimer 5G9 could recognize and bind to human EGFRs or mouse EGFRs on the surface of human breast cancer cells of the MDA-MB-231 cell line or mouse Lewis lung cancer cells (LLC), effectively inhibiting growth and inducing apoptosis in the targeted cells. This work lays the foundation for further studies investigating the feasibility of this strategy for targeting the dimer interface of EGFR and developing novel anti-EGFR drugs.
\end{abstract}

Keywords: EGFR; Dimerization; Dimer interface; Monoclonal antibody; Anti-cancer 


\section{Introduction}

The epidermal growth factor receptor (EGFR), a transmembrane receptor tyrosine kinase (RTK) and member of the ErbB (human epidermal receptor, HER)receptor family, plays an important role in the growth and differentiation of normal epidermal cells. EGFR contains an extracellular ligand binding domain, followed by a single transmembrane domain and a cytoplasmic domain containing a conserved protein tyrosine kinase (PTK) core flanked by regulatory sequences [1-3]. Upon ligand binding, the extracellular domain (ECD) of EGFR undergoes considerable conformational changes and a significant rearrangement that projects the dimerization arm outward to engage in receptor dimerization. Dimerization results in intrinsic PTK activation and tyrosine autophosphorylation, which initiates a cascade of intracellular mitogenic signaling and other cellular activities [4-7]. EGFR can be aberrantly activated by over-expression or mutations, resulting in cell proliferation, angiogenesis, metastasis, and anti-apoptosis. EGFR is over-expressed in a variety of epidermal cancers, such as head and neck, lung, colon, breast, ovarian, brain and pancreatic cancers, and it correlates with poor response to treatment, disease progression, and poor survival, making it an attractive therapeutic target for the treatment of epithelial cancers [8-10]. The dominant strategy for inhibiting EGFR is targeting the extracellular domain (ECD) with monoclonal antibodies (mAbs) or the intracellular domain with tyrosine kinase inhibitors (TKIs). Several EGFR antagonists have been approved, including the mAbs cetuximab (Erbitux) and panitumumab (Vectibix) and the TKIs gefitinib (IRESSA) and erlotinib (TARCEVA) $[11,12]$. These EGFR antagonists were shown to be effective and safe, causing only a reversible skin rash, when used in EGFR-positive patients individually or in combination with standard chemotherapeutics. However, the patients had a very low clinical response rate (approximately 10-30\%); furthermore, the patients frequently developed resistance to these antagonists [13, 14]. Although the causes and mechanisms are manifold, the polymorphism and the extensive mutations in the target sites are likely responsible for the low response rates of receptor antagonists, while heterodimerization is like the cause of the drug resistance. Therefore, 
targeting a more conservative site with an important function in the activation of EGFR may be a rational strategy for improving the response of anti-EGFR therapies.

The ECD of EGFR is composed of four subdomains, including domains I, II, III, and IV. Domains I and III form the ligand binding domain of EGFR, while domain II is responsible for its dimerization. When no ligand is bound, domain II is buried inside of the ECD and tethered by domain IV. Upon ligand binding to the cleft formed by Domains I and III on the surface of molecule, the tethered conformation undergoes a significant rearrangement that exposed the dimer interface and extended the dimerization arm beyond the other EGFR molecule (homodimerization) or the other receptor of the HER family (heterodimerization) $[15,16]$. The dimerization arm of human EGFR (hEGFR) located in the center of the dimer interface is a $\beta$-hairpin loop with a highly conservative sequence that spans residues 237-267 (EGFR237-267) and has $100 \%$ sequence homology with mouse EGFR (mEGFR). Since dimerization plays a crucial role in the activation of the HER receptor family, mAbs targeting the dimer interface of EGFR, especially the conservative $\beta$-hairpin loop directly participating in the dimerization, should effectively inhibit EGFR by preventing homodimerization or heterodimerization (as shown in Fig. 1). We have previously reported a chimeric peptide vaccination based on EGFR (237-267) and a "promiscuous" Th-cell epitope MVF from the measles virus fusion protein that could arouse specific immune response in mice; furthermore, peptide-specific antibodies could recognize and inhibit human or mouse epithelial cancer cells over-expressing EGFR [17]. To further explore the feasibility of the strategy of targeting the dimer interface of EGFR and to develop novel antibodies with better clinical responses, an EGFR beta-hairpin-specific mouse mAb was generated and studied in this paper.

\section{Materials and Methods}

\subsection{Mice}


6-8-week-old female BALB/c and Kunming mice were selected as animals for immunity and feeder, respectively. All animals were purchased from Guangdong Experimental Animal Center (China). Animal care and use was in accordance with institutional guidelines.

\subsection{Cell lines and culture medium}

Mouse myeloma Sp2/0 cells were a gift from Prof. Tian. The human breast cancer cell line MDA-MB-231 was purchased from Guangzhou Jennio Biotech Co., Ltd. (China). The mouse fibroblast cell line 3T3 was gifted from Guangzhou Institutes of Biomedicine and Health, Chinese Academy of Science. Lewis lung cancer cells (LLC) and human cervical carcinoma HeLa cells were purchased from the Chinese Academy Type Culture Collection. The molecular phenotypes of MDA-MB-231, LLC, 3 T3 and Hela cells were identified using western blotting to over-express hEGFR, over-express mEGFR, normally express mEGFR and be EGFR-negative, respectively. DMEM, RPMI-1640, fetal bovine serum (FBS), and supplements were purchased from Hyclone Life Technologies, Ltd. (USA).

\subsection{Preparation of antigen peptides}

The antigen peptides BSA-EGFR ${ }^{237-267}$ and OVA-EGFR ${ }^{237-267}$ were prepared with glutaraldehyde [18], and BSA and OVA were purchased from Sigma (USA). The B cell epitope peptide EGFR ${ }^{237-267}$ was synthesized by Shanghai Chutai Biotechnology Co., Ltd. (China).

\subsection{Immunization of animals and the titer determination of the antisera}

A humoral immune response against BSA-EGFR ${ }^{237-267}$ was induced by immunizing five female BALB/c mice, which were immunized subcutaneously with $0.2 \mathrm{mg}$ of peptide dissolved in double-distilled water (ddH2O) and emulsified (1:1) in 
Freund's complete adjuvant (Sigma, USA). Then, $0.2 \mathrm{mg}$ of peptide was dissolved in ddH2O and emulsified (1:1) in Freund's incomplete adjuvant at 2-week intervals. The blood was collected via the caudal vein and then centrifuged at 10,000 $\mathrm{g}$ for $10 \mathrm{~min}$ at $4^{\circ} \mathrm{C}$. The supernatants' titers were determined utilizing a previous method [17] using OVA-EGFR $^{237-267}$ as the coating antigen of a solid phase ELISA.

\subsection{Generation of hybridomas}

The mouse with the highest antiserum titer was selected for fusion. The spleen of this mouse was removed, and its splenocytes were fused with the mouse myeloma cell line SP2/0. Antibody-secreting cells were screened by ab ELISA using OVA-EGFR $^{237-267}$ as the coating antigen [19]. From 351 clones screened, 27 positive clones (readings were two times more than the negative control) were obtained in the initial screening. Among them, 16 clones were selected for the repeated screening. A total of 4 clones that could recognize the antigen were generated. Established hybridomas producing $\mathrm{mAbs}$ against the peptide $\mathrm{EGFR}^{237-267}$ were stored in liquid nitrogen.

\subsection{Preparation and purification of the monoclonal antibody}

$\mathrm{BALB} / \mathrm{c}$ mice that were 6 to 8 -weeks-old were injected intraperitoneally with 0.5 $\mathrm{ml}$ of mineral oil; then, the mice rested for 7 days before being injected intraperitoneally with approximately $1 \times 10^{6}$ positive hybridoma cells. Ascites fluids were collected daily, centrifuged at $10,000 \mathrm{~g}$ for 10 minutes, and stored in the presence of sodium azide at $4^{\circ} \mathrm{C}$. The supernatants of the ascites fluids were purified by the steric exclusion of polyethylene glycol [20]. Then, the crude products were diluted to 1 $\mathrm{mg} / \mathrm{ml}$ with a binding buffer containing $0.5 \mathrm{~mol} / \mathrm{L} \mathrm{NaCl}$ at $\mathrm{pH} 7.4$ and applied to a 
protein A agarose column (GE, USA) [21]. The bound protein was eluted using an elution buffer containing $0.1 \mathrm{~mol} / \mathrm{L}$ glycine at $\mathrm{pH} 2.7$. The buffer solution was permutated in $0.01 \mathrm{~mol} / \mathrm{L}$ PBS (pH 7.4) using Sephadex G-25 beads (GE, USA). The purity and molecular weight of the monoclonal antibodies were determined by sodium dodecyl sulfate polyacrylamide gel electrophoresis (SDS-PAGE). The titer and affinity was calculated using an indirect ELISA $[22,23]$ with OVA-EGFR ${ }^{237-267}$ as the coating antigen.

\subsection{Antibody isotyping}

The isotype and light chain of the mAb produced by the hybridoma cells were determined using an isotyping enzyme-linked immunosorbent assay kit (Sigma, USA) according to the manufacturer's instructions.

\subsection{The binding of the $\beta$-hairpin-specific mAb Antidimer $5 G 9$ to cells by Laser}

\section{Scanning Confocal Microscopy}

Cells were plated at $1 \times 10^{5} \mathrm{cell} / \mathrm{ml}$ on Petri dishes and cultured in DMEM supplemented with $10 \%$ (v/v) FBS for $24 \mathrm{~h}$. Then, the cells were washed twice with ice-cold PBS. The cells were fixed with $4 \%$ paraformaldehyde in PBS for 15 min and washed with PBS three times to quench the fixation. Then, the cells were blocked with $1 \%(w / v)$ BSA in PBS. Then, the cells were incubated with Antidimer 5G9 or cetuximab at $37{ }^{\circ} \mathrm{C}$ for $2 \mathrm{~h}$, which were treated with goat-anti-mouse or rabbit-anti-human FITC-conjugated Abs, respectively, for $1 \mathrm{~h}$ at room temperature. After washing, the cells were analyzed by Laser Scanning Confocal Microscopy.

\subsection{Assay of the $\beta$-hairpin-specific binding of the $m A b$ Antidimer $5 G 9$ to cells by} Flow Cytometry 
A total of $1 \times 10^{6}$ cells were incubated with the appropriate concentration of the indicated drug in $100 \mu \mathrm{l}$ of $2 \%(\mathrm{w} / \mathrm{v}) \mathrm{BSA}$ in PBS for $2 \mathrm{~h}$ at $4{ }^{\circ} \mathrm{C}$. Normal mouse immunoglobulin was used as a negative control. Unbound Abs were removed with PBS, and the cells were incubated with the goat-anti-mouse FITC-conjugated Abs for $30 \mathrm{~min}$ at $4{ }^{\circ} \mathrm{C}$ in $100 \mu \mathrm{l}$ of $2 \%(\mathrm{w} / \mathrm{v}) \mathrm{BSA}$ in PBS. Then, the cells were washed in 10 mmol/L PBS (pH 7.4) and fixed in $1 \%(\mathrm{v} / \mathrm{v})$ formaldehyde before being analyzed on a Coulter ELITE flow cytometry (Beckman, USA). Debris, cell clusters, and dead cells were gated out using light scatter assessment before single-parameter histograms were drawn and smoothed.

\subsection{MTT cell growth inhibition assay}

Cells were seeded onto 96-well plates (Costar) at 5,000 cells/well and incubated with $10 \%$ (v/v) FBS medium overnight. The medium was replaced with the appropriate concentration of the indicated drug. After incubating for 3 to 5 days at $37^{\circ} \mathrm{C}$, the cells were treated with MTT at $0.5 \mathrm{mg} / \mathrm{ml}$ for $4 \mathrm{~h}$ at $37^{\circ} \mathrm{C}$. The numbers of viable cells were measured using the bioconversion of MTT by reading the OD490. All experiments were performed in triplicate. The percentage of inhibition was calculated using the following formula: (ODnormal mouse IgG - OD anti- $\beta$-hairpin mAb)/OD normal mouse $\operatorname{IgG} \times 100$.

2.12. Assay of the induction of apoptosis by the $\beta$-hairpin specific mAb Antidimer $5 G 9$ using Flow Cytometry

MDA-MB-231 cells were seeded onto six-well culture plates at $3 \times 10^{5}$ cells/well and incubated overnight at $37{ }^{\circ} \mathrm{C}$ in a $5 \% \mathrm{CO}_{2}$ incubator. Subsequently, the cells were treated with Antidimer 5G9 (10, 20, or $80 \mu \mathrm{g} / \mathrm{mL})$ or cetuximab $(10,20$, or $80 \mu \mathrm{g} / \mathrm{mL})$. The control cells were treated with PBS. All the cells were incubated for $72 \mathrm{~h}$ at $37{ }^{\circ} \mathrm{C}$ 
in a $5 \% \mathrm{CO}_{2}$ incubator. After treatment, the cells were trypsinized, washed with PBS, and resuspended in $100 \mu \mathrm{L}$ of binding buffer (50 mM HEPES/NaOH, pH 7.4, $700 \mathrm{mM}$ $\mathrm{NaCl}$, and $12.5 \mathrm{mM} \mathrm{CaCl}_{2}$ ) containing $5 \mu \mathrm{L}$ of an Annexin V-FITC stock solution (Invitrogen, Paisley, UK) and $1 \mu \mathrm{L}$ of $1 \mathrm{mg} / \mathrm{mL}$ PI (Sigma, USA). The cells were incubated in a dark at room temperature for $15 \mathrm{~min}$, and another $400 \mu \mathrm{L}$ of binding buffer was added. The samples were quantified by flow cytometry using a FACSCanto II (Beckman, USA).

\subsection{Statistical analysis}

The data were reported as the mean \pm standard deviation. Each experiment was subjected to statistical analysis using the Student-Newmann-Keuls analysis of variance and t-test for grouped data. Significance was accepted as a $P$ less than or equal to 0.05 .

\section{Results}

\subsection{Generation and preparation of the $\beta$-hairpin specific $m A b$}

The peptide EGFR ${ }^{237-267}$, a highly conserved $\beta$-hairpin loop sequence, was prepared using solid phase organic synthesis. Furthermore, the coupling peptide BSA-EGFR $^{237-267}$ was selected as the immunogen, and OVA-EGFR ${ }^{237-267}$ was used as the coating antigen in the ELISAs completed. After three injections, over $1 \times 10^{4}$ titers of peptide-specific Abs were elicited in mice (shown as Fig. 2). Four clones secreting high affinity peptide-specific mAbs were screened out from the hybridoma cells; three of which produced $\mathrm{IgG} 1$ isotypes of $\mathrm{mAbs}$ with affinity constants over $1 \times 10^{9} \mathrm{~L} / \mathrm{mol}$ (shown as Table 1). The mAb with the highest titer and affinity, named Antidimer 5G9, was selected for further tests. Antidimer 5G9 was purified from ascites fluids by the steric exclusion of polyethylene glycol and protein A agarose affinity chromatography, which showed two characteristic bands and over $95 \%$ purity in the SDS-PAGE analysis (shown as Fig. 3). 


\subsection{The binding of the $\beta$-hairpin specific $m A b$ Antidimer $5 G 9$ to over-expressing EGFR cells}

Since the amino acid sequences of the $\beta$-hairpins between hEGFR and mEGFR are $100 \%$ homologous, the $\beta$-hairpin peptide-specific mAb Antidimer 5G9 should recognize and bind to both EGFRs on the surface of cells. To test the specific binding capacity of Antidimer 5G9, the human breast cancer cell line MDA-MB-231 (over-expressing hEGFR) and the murine lung cancer cell (LLC, over-expressing mEGFR) were used for laser scanning confocal microscopy analysis. The confocal analysis showed that a large number of Antidimer 5G9 bound to the surface of both cell types, but the humanized mAb cetuximab, which targets the hEGFR's ligand-binding site, almost did not bind to the surface of LLC cells at all (shown as Fig. 4a). These results implied that Antidimer 5G9 can recognize the $\beta$-hairpins of native hEGFR and mEGFR on the surface of the cells tested here. Additionally, flow cytometry analysis with MDA-MB-231 cells showed that the binding effect of Antidimer 5G9 was dose-dependent (shown as Fig. 4b), which further confirmed the specificity of its binding.

\subsection{Anti-proliferative effects of the $\beta$-hairpin-specific mAb Antidimer $5 G 9$}

The effects of the mAb Antidimer 5G9 on the proliferation of different cell lines were examined using a MTT assay, in which different EGFR-phenotype cells were used. The purified Antidimer 5G9 had significant anti-proliferative effects on over-expressing hEGFR MDA-MB-231 and over-expressing mEGFR LLC cells. The inhibition ratios were over $60 \%$ and $40 \%$, respectively, but the purified Antidimer 5G9 had few effects on the inhibition ratios of mouse fibroblast 3T3 cells (normally expressing mEGFR) and human cervical carcinoma HeLa cells (EGFR negative) 
(shown as Fig. 5). Furthermore, our tests showed that the anti-proliferative effect of Antidimer 5G9 was dose-dependent and its half-maximal inhibitory concentration (IC50) of $9.55 \mu \mathrm{g} / \mathrm{ml}$ was slightly lower than that of cetuximab $(12.88 \mu \mathrm{g} / \mathrm{ml})$.

\subsection{Antidimer $5 G 9$ induces apoptosis in cells over-expressing EGFR}

To quantify the percentage of apoptotic cells, we performed an Annexin V-FITC and PI double staining assay and then analyzed the results by flow cytometry. The MDA-MB-231 cells were treated with different doses of Antidimer 5G9 or cetuximab for 72 h. As shown in Fig. 6a, a total of $37.68 \%, 54.0 \%$, and $81.6 \%$ of the MDA-MB-231 cells were in the apoptotic phase (B2: late apoptotic, + B4: early apoptotic) after being treated with Antidimer 5G9 at 10, 20, and $80 \mu \mathrm{g} / \mathrm{mL}$, respectively, which showed that Antidimer 5G9 induced apoptosis in a dose-dependent manner. Furthermore, a total of $21.1 \%, 36.5 \%$, and $48.0 \%$ of the cells treated with cetuximab underwent apoptosis. In addition, the cells treated with Antidimer 5G9 exhibited a higher total apoptosis rate than that those treated with cetuximab at the same dose (shown as Fig. 6b). Based on this finding, we concluded that Antidimer 5G9 was more potent than cetuximab in inducing cell apoptosis.

\section{Discussion}

The hypermutation of the extracellular domain and the intracellular domain and the heterodimerization of EGFR are the main causes for the low clinical response and resistance of EGFR antagonists $[13,14]$. Therefore, targeting the highly conserved $\beta$-hairpin of EGFR, which directly participates in the homodimerization or heterodimerization of the receptor, is a reasonable optimization strategy for the development of anti-EGFR drugs. To evaluate the feasibility of targeting and developing a novel therapeutic $\mathrm{mAb}$, a murine $\mathrm{mAb}$ was needed. Since the amino acid sequences of the $\beta$-hairpin of hEGFR and mEGFR are $100 \%$ homologous, it is important to determine whether the antigen peptide from the homologous $\beta$-hairpin can break the immune tolerance of mice. Just as our preliminary study reported that a 
chimeric antigen composed of EGFR ${ }^{237-267}$ and the universal Th-epitope peptide MVF did [17], the coupling antigen of EGFR ${ }^{237-267}$ and BSA could effectively excite an intense peptide-specific immune response. Three lines of IgG1-like EGFR ${ }^{237-267}$ -specific mAbs with affinity constants over $1 \times 10^{9} \mathrm{~L} / \mathrm{mol}$ were successfully generated, of which Antidimer 5G9 was tested for its specific capacities.

Unlike cetuximab, as a chimeric monoclonal antibody binding to the ligand-binding domain on the surface of EGFR in ligand-competitive fashion, Antidimer 5G9 should bind to the $\beta$-hairpin ligand-dependently because the exposure of the dimer interface needs ligand-binding. Antidimer 5G9 could bind in abundance to the surface of both over-expressing hEGFR cells (MDA-MB-231) and over-expressing mEGFR cells (LLC), suggesting that this mAb could effectively recognize and bind to the $\beta$-hairpin of EGFR on the surface of the cells as designed. Our preliminary work also showed that the binding of the EGFR ${ }^{237-267}$-specific polyclonal antibodies to the surface of the cells was ligand-dependent [17]. Antidimer 5G9 showed more powerful proliferation-inhibiting and apoptosis-inducing activities than cetuximab, which should be credited to the ligand-dependence of the binding of this novel mAb to EGFR and its direct blocking effects on the dimerization. However, whether Antidimer 5G9 can effectively inhibit the dimerization, particularly the heterodimerization, of EGFRs on the surface of cells needs further investigation. On the other hand, it is also important to determine whether Antidimer 5G9 can induce the endocytosis of EGFRs on the surface of cells, as well as antibody-dependent cell-mediated cytotoxicity (ADCC) and complement-dependent cytotoxicity (CDC), as cetuximab did. The ability of Antidimer 5G9 to recognize the EGFRs of both humans and mice can facilitate the study of its pharmacodynamics. A series of animal xenografts preferably mimicking the clinical status, such as tumor cells of different EGFR phenotypes and dimerization patterns, 
will be employed to evaluate the feasibility of this strategy for anti-EGFR therapy by targeting the dimerization and the potential of Antidimer 5G9 for the design of novel therapeutic mAbs.

\section{Conclusions}

In summary, we present herein a novel monoclonal antibody targeting the $\beta$-hairpin loop on the dimer interface of EGFR. Our observations could possibly open novel therapeutic options for EGFR-over-expressing tumors.

\section{Conflict of interests}

The authors declare that they have no conflict of interest.

\section{Acknowledgments}

This work was supported by the National Science Foundation of China (No. 81273423) and the Guangdong Science and Technology Plan Project (2014A020210027, 2015A030310120).

\section{References}

[1] Thompson DM, Gill GN. The EGF receptor: structure, regulation, and potential role in malignancy. Cancer Surv 1985; 4:767-88.

[2] Roskoski Jr R. The ErbB/HER family of protein-tyrosine kinases and cancer. Pharmacol Res 2014; 79:34-74.

[3] Lemmon MA, Schlessinger J. Cell signaling by receptor tyrosine kinases. Cell 2010; 141:1117-34.

[4] Schlessinger J. Ligand-induced, receptor-mediated dimerization and activation of EGF receptor. Cell 2002; 110:669-72. 
[5] Liu P, Cleveland 4th TE, Bouyain S, Byrne PO, Longo PA, Leahy DJ. A single ligand is sufficient to activate EGFR dimers. Proc Natl Acad Sci USA 2012; 109:1086-96.

[6] Olayioye MA, Neve RM, Lane HA, Hynes NE. The ErbB signaling network: receptor heterodimerization in development and cancer. The EMBO journal. 2000; 19(13:3159-3167.

[7] Ocaña A, Pandiella A.Targeting HER receptors in cancer. Curr Pharm Des. 2013; 19(5):808-17.

[8] Reardon DA, Wen PY, Mellinghoff IK. Targeted molecular therapies against epidermal growth factor receptor: past experiences and challenges. Neuro Oncol 2014; 16 Suppl 8:viii7-13.

[9] SeshacharyuluP, PonnusamyMP, HaridasD, JainM, GantiAK, BatraSK. Targeting the EGFR signaling pathway in cancer therapy. Expert Opin Ther Targets 2012;16:15-31.

[10] Capdevila J, Elez E, Macarulla T, Ramos FJ, Ruiz-Echarri M, Tabernero J. Anti -epidermal growth factor receptor monoclonal antibodies in cancer treatment. Cancer Treatment Reviews 2009; 35(4):354-63.

[11] Gasser M, Waaga-Gasser AM. Therapeutic Antibodies in Cancer therapy. Adv Exp Med Biol 2016; 917:95-120.

[12] Sin TK, Wang F, Meng F, Wong SC, Cho WC, Siu PM, Chan LW, Yung BY. Implications of MicroRNAs in the Treatment ofGefitinib-Resistant Non-Small Cell Lung Cancer. Int J Mol Sci 2016; 17(2):237. 
[13] Curtis R Chong, Pasi A Jänne. The quest to overcome resistance to EGFR-targeted therapies incancer. Nat Med 2013; 19(11): 1389-1400.

[14] Gorjan Hrustanovic, Bianca J. Lee, Trever G. Bivona. Mechanisms of resistance to EGFR targeted therapies. Cancer Biol Ther 2013;14(4): 304-314.

[15] Olayioye MA, Neve RM, Lane HA, Hynes NE. The ErbB signaling network: receptor heterodimerization in development and cancer. The EMBO journal. 2000; 19(13:3159-3167.

[16] Morgillo F, Woo JK, Kim ES, Hong WK, Lee HY. Heterodimerization of insulin-like growth factor receptor/epidermal growth factor receptor and induction of survivin expression counteract the antitumor action of erlotinib. Cancer research. 2006; 66(20:10100-10111.

[17] Lei Zhu, Lin Zhao, Meizhi Wu, Zhange Chen, Huangjin Li. B-cell epitope peptide vaccination targeting dimer interface of epidermal growth factor receptor(EGFR). Immunology Letters 2013; 153(2013)33-40.

[18] Wang M, Shi H, Wu D, Han H, Zhang J, Xing Z, Wang S, Li Q. Glutaraldehyde cross-linking of immobilized thermophilic esterase on hydrophobic macroporous resin for application in poly( $\varepsilon$-caprolactone) synthesis. Molecules. 2014, 19(7):9838-49.

[19] Riaz A, Zhao X, Dai X, Li W, Liu L, Wan H, Yu Y, Wang L, Zhou Q. Mouse cloning and somatic cell reprogramming using electrofused blastomeres. Cell Res. 2011 May; 21(5):770-8. 
[20] Gagnon P, Toh P, Lee J. High productivity purification of immunoglobulin G monoclonal antibodies on starch-coated magnetic nanoparticles by steric exclusion of polyethylene glycol. J Chromatogr A 2014;1324:171-80.

[21] Zhang Q, Goetze AM, Cui H, Wylie J, Tillotson B, Hewig A, Hall MP, Flynn GC. Characterization of the co-elution of host cell proteins with monoclonal antibodiesduring protein A purification. Biotechnol Prog. Biotechnol Prog 2016; 32(3):708-17.

[22] Manoj J, Agarwal RK, Sailo B, Wani MA, Singh MK.Evaluation of recombinant outer membrane protein $\mathrm{C}$ based indirect enzyme-linked immunoassay for the detection of Salmonella antibodies in poultry.Vet World. 2015 Aug; 8(8):1006-10.

[23] Dogra V, Verma S, Singh G, Wani AH, Chahota R, Dhar P, Verma L, Sharma M. Development of OMP based indirect ELISA to gauge the antibody titers in bovines against Pasteurella multocida. Iran J Vet Res. 2015 Fall; 16(4):350-6.

\section{Figure legends}

Fig. 1 A schematic drawing of the strategy for targeting the dimer interface of EGFR.

Fig. 2 Five mice were immunized with BSA-EGFR ${ }^{237-267}$ for a total of three injections. Blood was drawn a week after every injection, and the sera were analyzed for anti-EGFR ${ }^{237-267}$ antibodies (Abs) by ELISA. Each bar represents one animal. Titers are defined as the reciprocal of the highest serum dilution with an absorbance of 0.2 or greater after subtracting the background. $1 \mathrm{I}+1 \mathrm{w}$ indicated the Abs titer in blood investigated 1 week (1w) after the first injection and so forth. 
Fig. 3 Analysis of Antidimer 5G9 purification. Lane 1 indicates the ascites fluid of the BALB/c mice. Lane 2 indicates Antidimer 5G9 after the first purification by the steric exclusion of polyethylene glycol. Lane 3 indicates the consummate Antidimer 5G9 purified by the protein A agarose column.

Fig. 4a The binding of the anti- $\beta$-hairpin $\mathrm{mAb}$ was detected by laser scanning confocal microscopy. (A), (B) and (C) show HeLa cells (EGFR negative, used as the control), MDA-MB-231 cells (over-expressing human EGFR) and LLC cells (over-expressing mouse EGFR), respectively, treated with Antidimer 5G9. (D), (E) and (F) show HeLa cells, MDA-MB-231 cells and LLC cells, respectively, treated with cetuximab.

Fig. 4b Effects of the concentration of $\mathrm{mAb}$ on the binding capability of Antidimer 5G9 to MDA-MB-231 cells. Flow cytometry was used to assess the binding capabilities of the mAb to the native receptor on MDA-MB-231 cells. The specific binding was detected with a FITC-labeled goat-anti-mouse secondary Abs. The x-axis represents the fluorescent intensity, and the y-axis represents the relative cell number. The red histogram indicated the normal mouse Immunoglobulin (negative). The rufous green, and blue histograms indicated cells treated with $0.05 \mathrm{mg} / \mathrm{ml}, 0.5 \mathrm{mg} / \mathrm{ml}$, and 5 $\mathrm{mg} / \mathrm{ml}$ purified Antidimer 5G9.

Fig. 5 Bioconversion of MTT was used to estimate the number of viable tumor cells remaining after 3 days. The proliferation inhibition rate was calculated using the following formula: (OD normal mouse IgG - OD anti- $\beta$-hairpin mAb)/OD normal mouse $\operatorname{IgG} \times 100$. The height of the column indicates the proliferation inhibition rate. Error bars represent \pm SEM. Left, inhibition of different cell lines; right, inhibition of MDA-MB-231 cells by a different doses of Antidimer 5G9 and cetuximab. The data 
were reported as the mean \pm standard deviation and were subjected to statistical analysis by Student-Newmann-Keuls analysis of variance. ${ }^{*}=p<0.05, * *=p<0.01$.

Fig. 6a Apoptotic death of MDA-MB-231 cells induced by Antidimer 5G9 and cetuximab as examined by an Annexin V-FITC/PI assay. MDA-MB-231 cells were treated with different concentrations of antibody for $48 \mathrm{~h}$. The percentages of cells in each quadrant are shown (B2: late apoptotic or necrotic cells, B3: viable cells, B4: early apoptotic cells). Letter a represents the cells treated with PBS. Letters b, c and d represent the cells treated with Antidimer 5G9 at $10 \mu \mathrm{g} / \mathrm{mL}, 20 \mu \mathrm{g} / \mathrm{mL}$ and $80 \mu \mathrm{g} / \mathrm{mL}$, respectively. Letters e, f and g represent the cells treated with cetuximab at $10 \mu \mathrm{g} / \mathrm{mg}$, $20 \mu \mathrm{g} / \mathrm{mL}$ and $80 \mu \mathrm{g} / \mathrm{mL}$, respectively.

Fig. 6b Statistical analysis of the total apoptotic rate of different groups at the same dose. The data are reported as the mean \pm standard deviation and were subjected to statistical analysis by Student-Newmann-Keuls analysis of variance. $*=p<0.05$, ** $=p<0.01$. 


\begin{tabular}{cccc}
\hline $\mathrm{mAb}$ & Titers & Affinity & isotype \\
\hline $3 \mathrm{E} 7$ & 1280000 & $1.745 \times 10^{9}$ & $\operatorname{IgG} 1$ \\
$3 \mathrm{~B} 12$ & 160000 & $1.009 \times 10^{9}$ & $\operatorname{IgG1}$ \\
$5 \mathrm{E} 9$ & 1280000 & $7.150 \times 10^{8}$ & $\operatorname{IgG} 2 \mathrm{~b}$ \\
$5 \mathrm{G} 9$ & 2560000 & $1.837 \times 10^{9}$ & $\operatorname{IgG1}$ \\
\hline
\end{tabular}

Table 1.The determine of titers, affinity, isotype of different anti- $\beta$-hairpin mAbs produced by the hybridoma cell lines. 


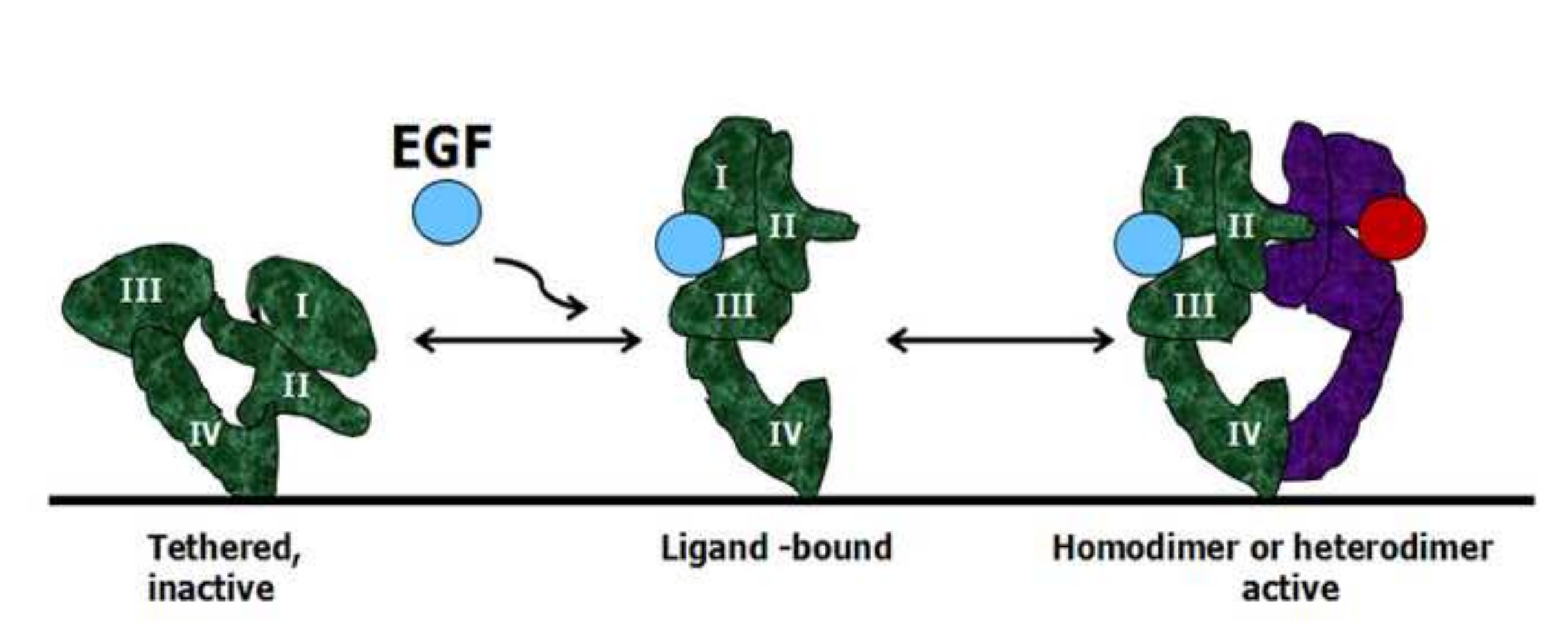

Tethered,
inactive

Tethered,
inactive

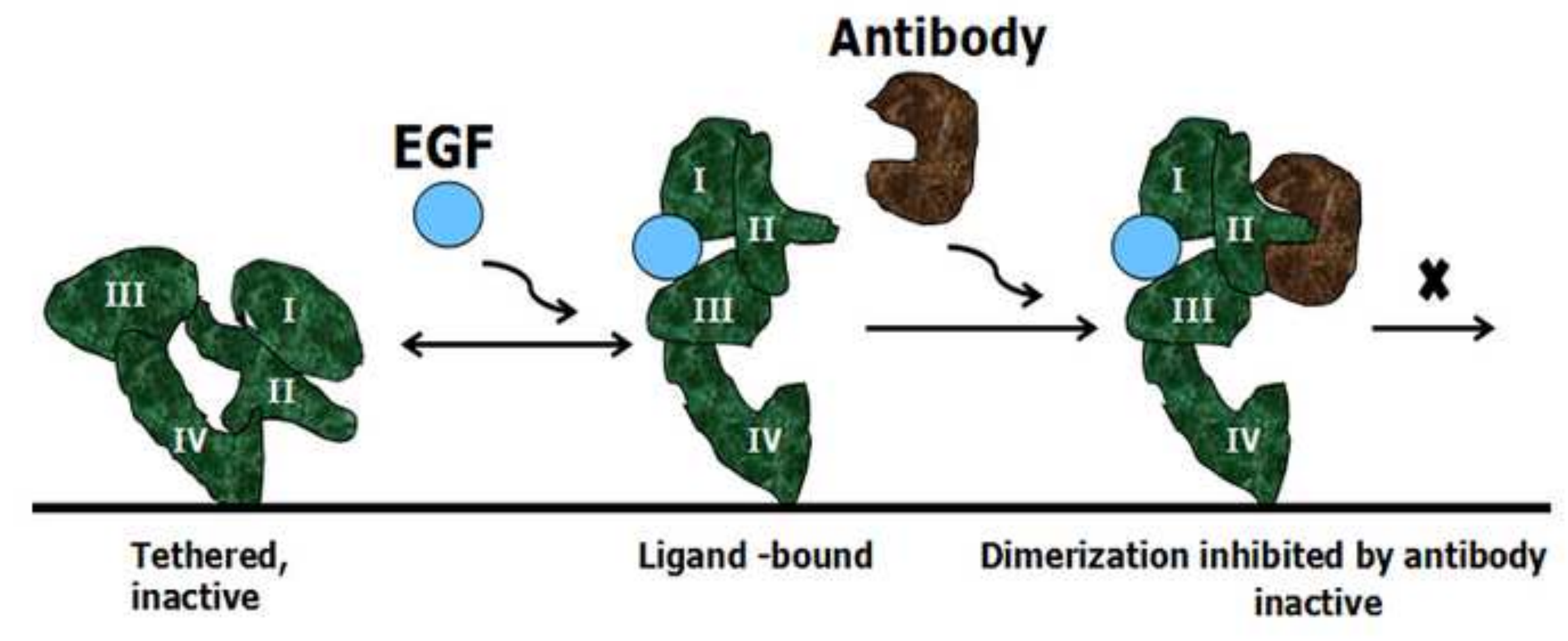
Tethered, inactive

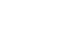

Ligand -bound

EGF

Ligand -bound

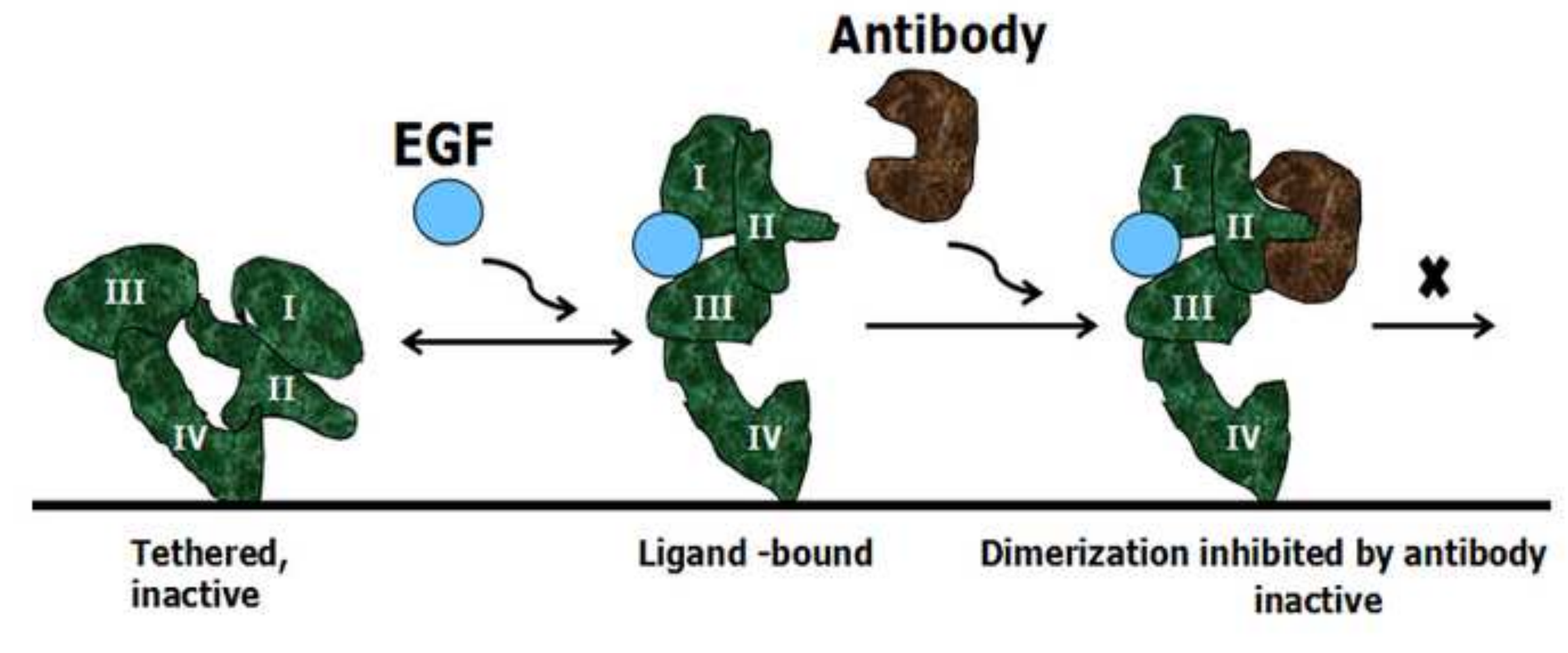

Homodimer or heterodimer active inactive

\author{
inactive
}

Antibody

Dimerization inhibited by antibody

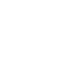




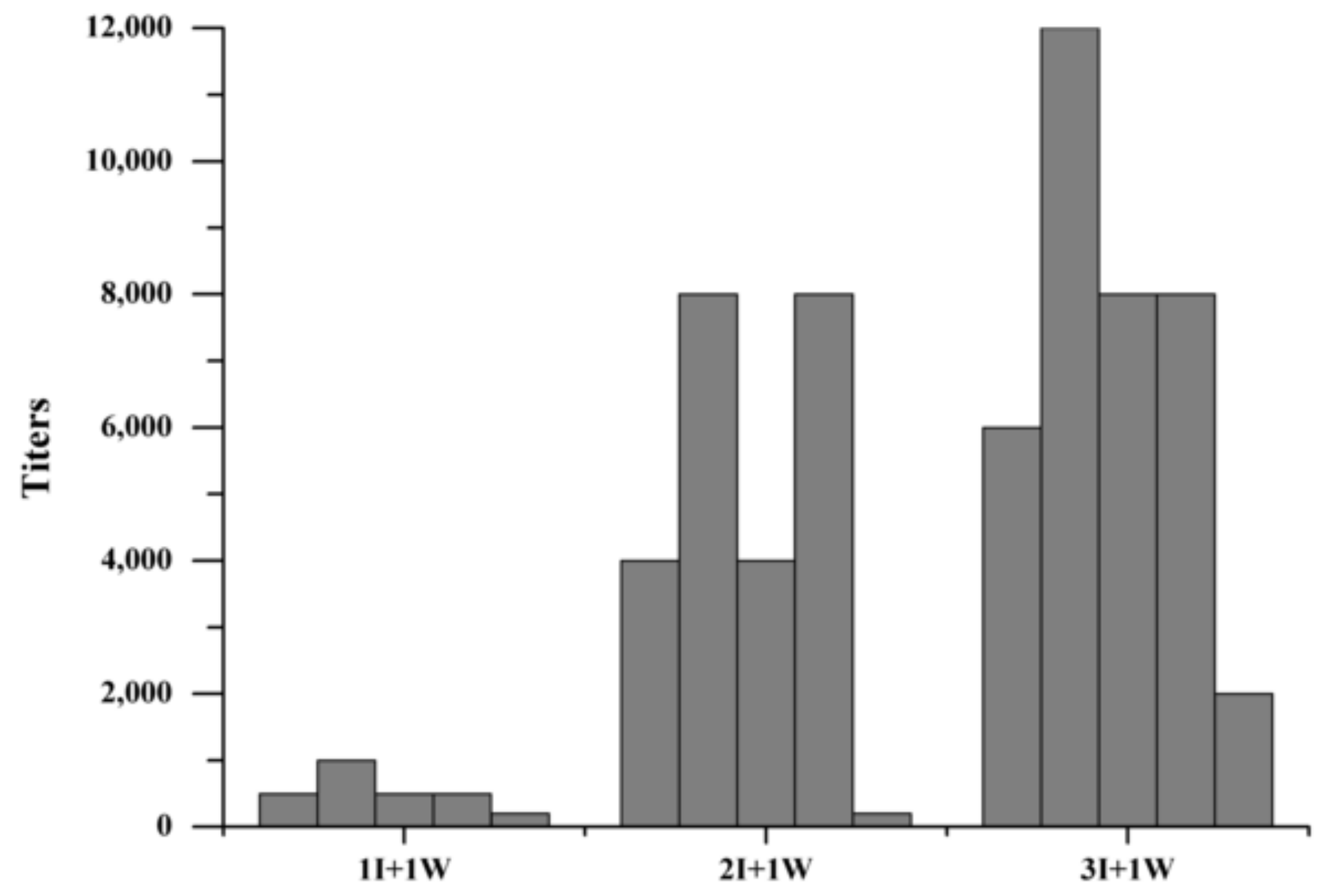

Time Post Immunization 


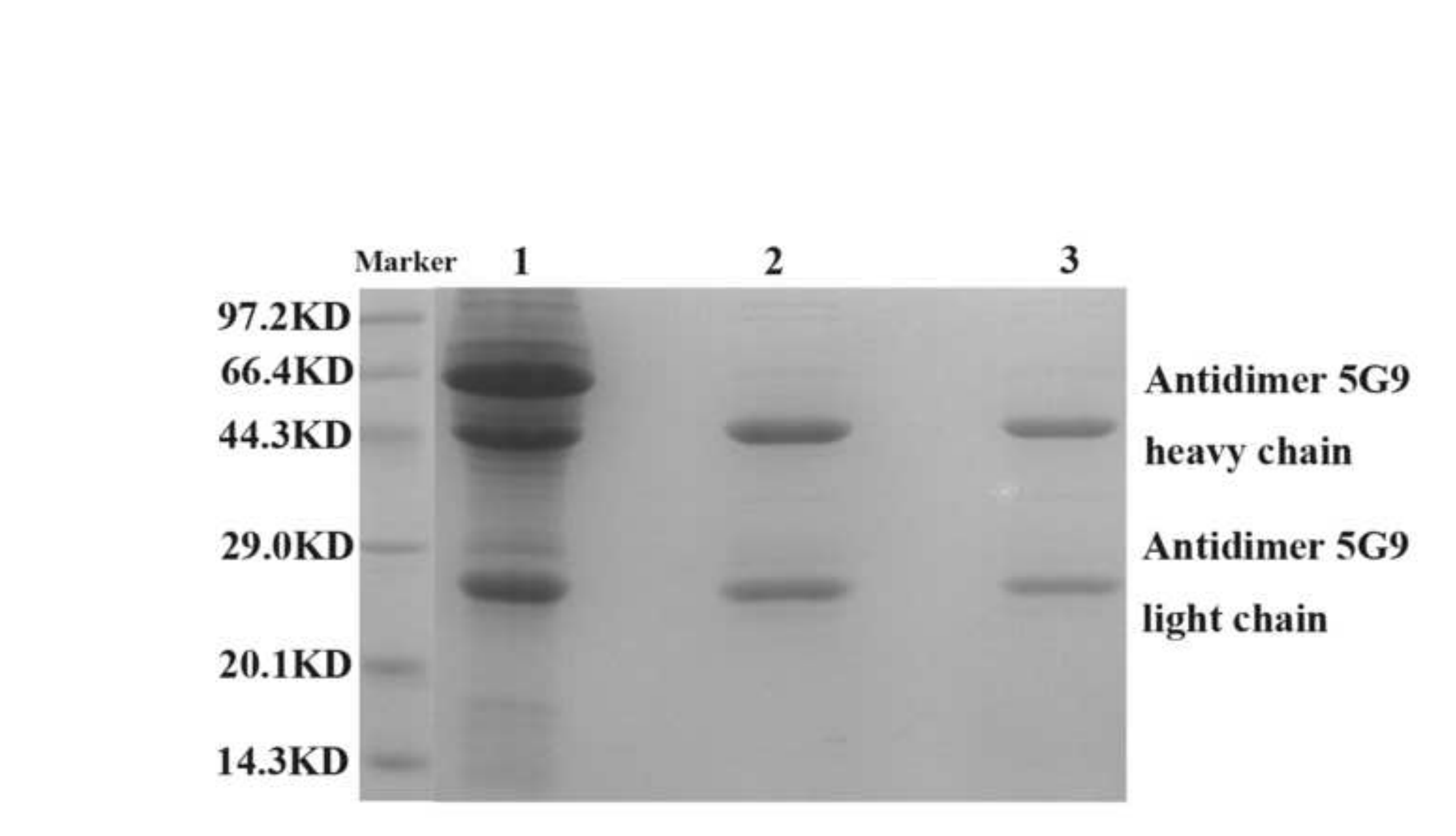

Antidimer 5G9

heavy chain

Antidimer 5G9

light chain

97.2KD

$66.4 \mathrm{KD}$

20.1KD

14.3KD

29.0KD 


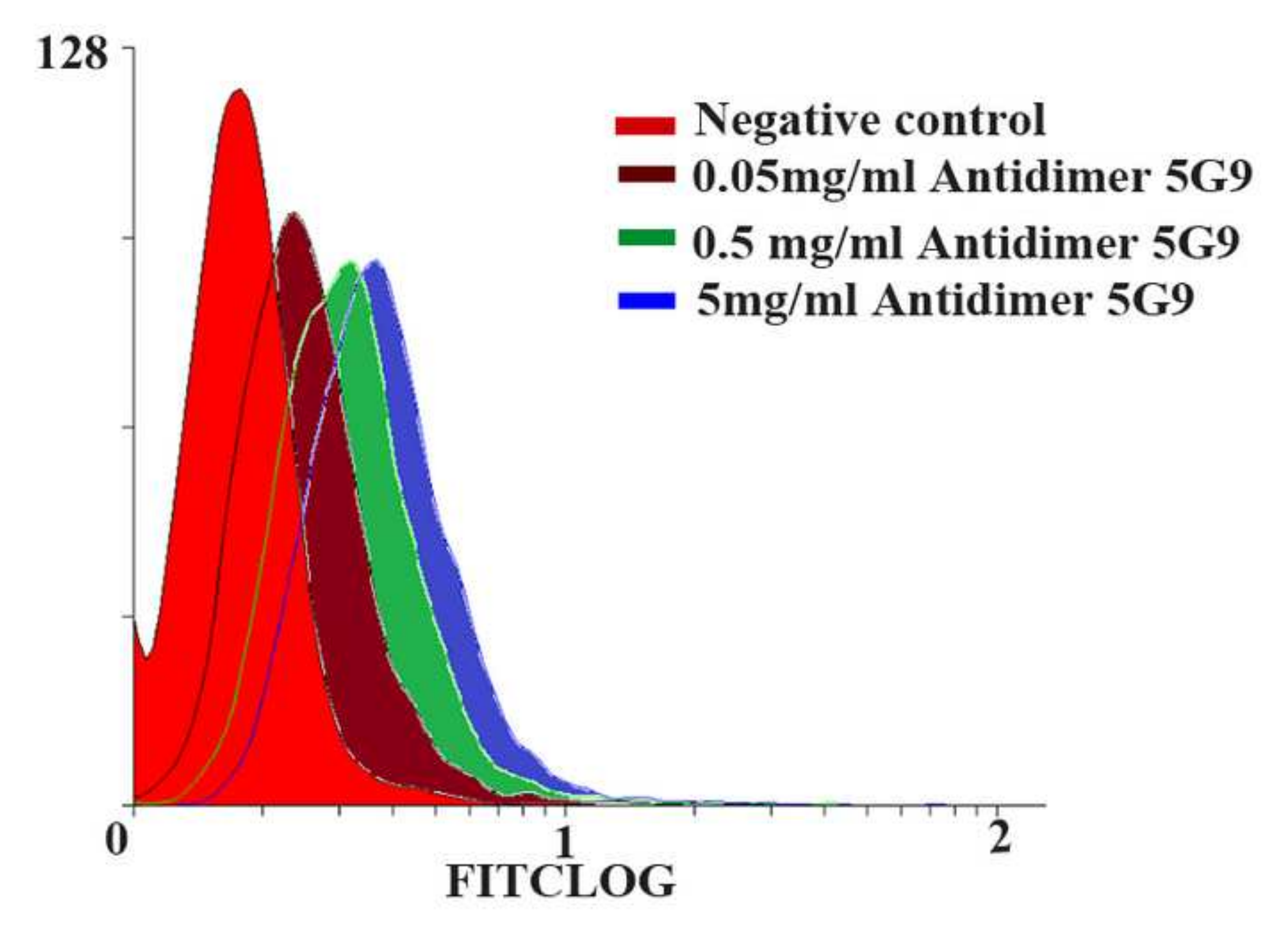

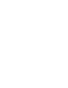

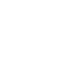

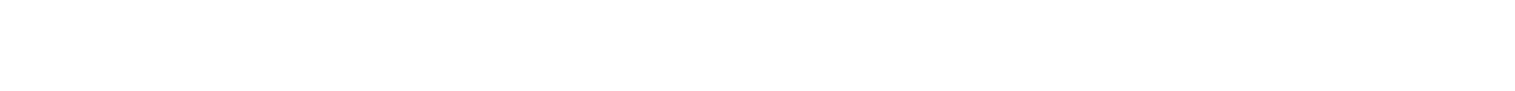



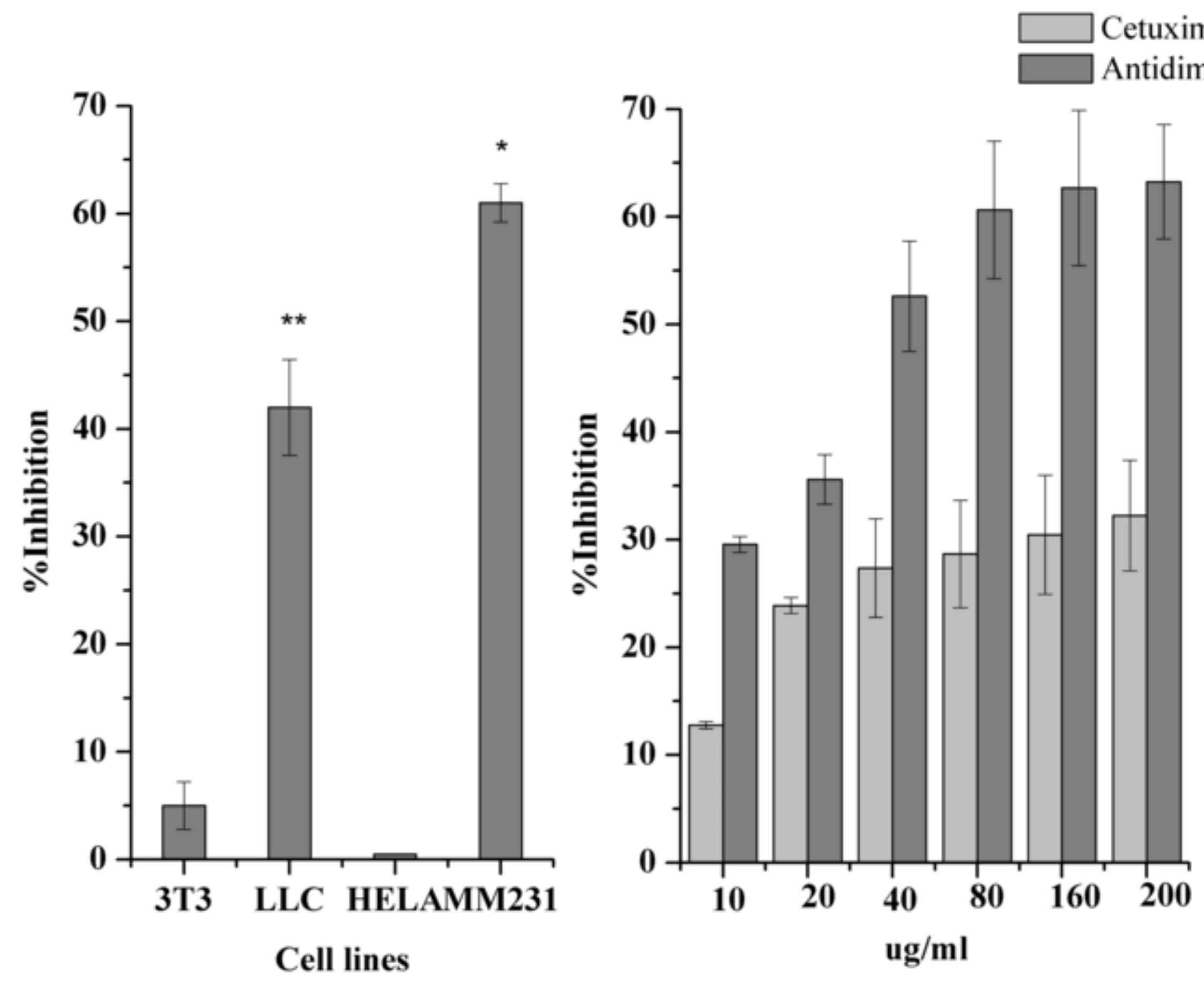

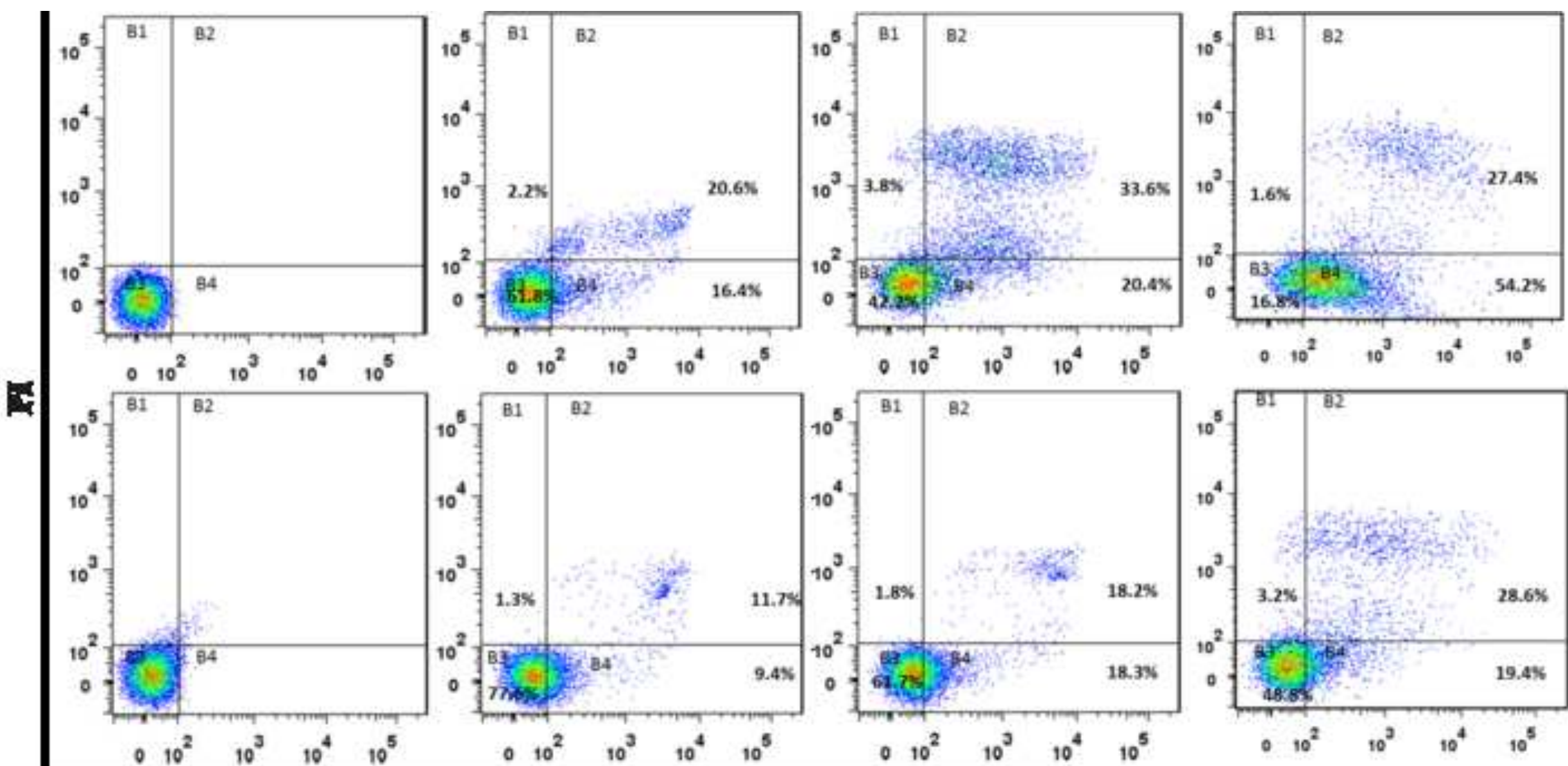

Aned V-MIC 
